\title{
Synthesis and Characterisation of Lamellar ZnS Nanosheets Containing Intercalated Diamines.
}

\author{
Shani Sperinck, Thomas Becker, Kate Wright and William R. Richmond* \\ Nanochemistry Research Institute, Department of Applied Chemistry, Curtin University \\ of Technology, GPO Box U1987, Perth WA 6845, Australia
}

*Corresponding author. E-mail: w.richmond@curtin.edu.au

\begin{abstract}
A solvothermal method has been used to prepare hybrid inorganic-organic composites with a lamellar structure in which layers of wurtzite ZnS are separated by intercalated diamine molecules. A hybrid composite prepared with diethylenetriamine has been isolated and characterised and its structure and properties compared with those of the composite prepared using ethylenediamine. Comparative structural and morphological studies of the two lamellar hybrid composites are described on the basis of powder XRD, electron and scanning probe microscopies and thermal analysis of the materials.
\end{abstract}

\section{Keywords}

ZnS Nanosheets, Layered compounds, Inorganic-organic hybrid composites, X-ray Diffraction, Atomic Force Microscopy.

\section{Introduction}

The miniaturization of electronic devices to the nanoscale level can benefit from the investigation of composite materials that combine the semiconducting functionality of an inorganic constituent with the lower weight and volume of an organic component. The organic component can be used to tailor the electronic properties of the inorganic framework by defining its reduced dimensionality and by mediating the electronic coupling between the inorganic units.[1] Rigidity and stability, in addition to superior electronic, magnetic, and optical properties, have made these inorganic frameworks highly attractive in the search for new, functional materials, while organic and coordination compounds built upon molecular building-blocks hold great promise for processability, flexibility, structural diversity, and geometrical control, including size, 
shape, and symmetry.[1, 2] Incorporation of the two counterparts into a single structure may generate inorganic-organic hybrid composites that enhance or combine the useful properties of both components.

Semiconductor nanoparticles have been studied intensely for many years because of their novel optical and transport properties which have great potential for many optoelectronic applications. Zinc sulfide belongs to the group II-VI semiconductors, and has been studied extensively for its use in optical coatings, solid-state solar windows,[3] thin film electroluminescent devices, non-linear optical devices, and as a flat-panel display when doped with divalent manganese ions.[3, 4] Synthetic approaches for these materials have included physical methods, such as thermal evaporation,[4, 5] vacuum sputtering and molecular beam epitaxy,[6] and chemical methods such precipitation in reverse micelles.[7, 8]

Of particular interest is the use of certain solvothermal methods to synthesize $\mathrm{ZnS}$ nanoparticles with highly anisotropic shapes[8] and the ability of these methods to produce the wurtzite phase at temperatures far below the $1020^{\circ} \mathrm{C}$ normally required for the phase change.[9] Depending on the synthesis environment, the morphology, size and crystallinity of the particles can vary quite dramatically. The use of ethylenediamine (en) as a solvent has been found to have a significant effect on the shape and morphology of the $\mathrm{ZnS}$ nanostructures.[3, 5, 10-14] It acts as a molecular template to direct the crystal structure and morphology of the resulting particles, which are square or rectangular micron-sized sheets of wurtzite-phase $\mathrm{ZnS}$.

This unique morphology has been shown to arise through formation of an inorganic-organic hybrid composite in which $\mathrm{ZnS}$ lamellae are interconnected by ethylenediamine molecules, each bridged to two Zn metal atoms in adjacent layers.[1012] Inorganic-organic hybrids of this type have been reported demonstrating ethylenediamine inclusion in various chalcogenide lamellar phases including ZnS,[15] ZnSe,[12, 16, 17] MnSe,[16] and ZnTe,[2] but the studies of these materials have been largely concerned with their role as precursors to the formation of thin-layer semiconductor particles. When heated in an inert atmosphere, the intercalated ethylenediamine is removed at $\sim 350^{\circ} \mathrm{C}$ to yield wurtzite $\mathrm{ZnS}$ nanosheets. Analogous inorganic-organic hybrids incorporating 1,3-propanediamine (pda) have also been prepared in lamellar ZnSe,[16, 17] MnSe[16] and ZnTe[2] composites, and their thermal decompositions behaviour is similar to that of the ethylenediamine compounds.[16] 
The factors governing formation of the layered ethylenediamine intermediates have not yet been well described and the mechanism by which they form has not been closely investigated. Furthermore, very little has been reported on the formation of hybrid composites with intercalated diamine molecules other than ethylenediamine or propylenediamine. These unique layered materials provide an ideal opportunity to precisely tailor the spacings between semiconductor thin layer within an inorganicorganic composite, or if the surfaces of these platelet composites can be properly characterised, to control at the molecular level the arrangement of organic moieties on a semiconductor surface. This may offer exciting possibilities for applications such as nanoscale molecular sensor technologies and optical devices.

Herein, we report the solvothermal preparation and characterisation of a novel composite organic-inorganic hybrid chalcogenide prepared in diethylenetriamine (deta) ZnS(deta) 0.5 . Characterisation of this phase is described along with some new morphological and surface characterisation of the related layered composite formed in ethylenediamine (en).

\section{Experimental}

\section{Synthesis}

All reactions were carried out in sealed Teflon-lined autoclaves of $23 \mathrm{ml}$ capacity. Zinc Acetate $\left[\mathrm{Zn}\left(\mathrm{CH}_{3} \mathrm{COO}\right)_{2} \cdot 2 \mathrm{H}_{2} \mathrm{O}\right](3 \mathrm{mmol})$ and Thiourea $\left[\mathrm{SC}\left(\mathrm{NH}_{2}\right)_{2}\right](7 \mathrm{mmol})$ were added to $15 \mathrm{ml}$ of alkylamine solvent (ethylenediamine or diethylenetriamine). The vessel was sealed, placed in an oven and maintained at a temperature of $180^{\circ} \mathrm{C}$ for 24 hours, then allowed to cool naturally to room temperature. The solution was filtered and the product was collected by centrifugation. The resulting white powders were thoroughly washed with ethanol in repeated centrifugation/resuspension cycles, then dried in an oven at $60^{\circ} \mathrm{C}$ over two days.

Phase-pure $\mathrm{ZnS}(\mathrm{en})_{0.5}$ was obtained in $>90 \%$ yield when ethylenediamine was used as the reactive solvent. ZnSCNH 4 Calc. C, 9.42; H, 3.16; N, 10.99; Zn, 51.28; Exp. C, 9.76; H, 3.38; N, 11.35; Zn, 51.51. Use of diethylenetriamine as the reactive solvent apparently did not yield a single-phase product, but elemental analysis was consistent with a phase mixture containing predominantly $\mathrm{ZnS}(\text { deta })_{0.5}$ with a small amount of 
excess ZnS. Calc. $\mathrm{ZnS}(\text { deta) })_{0.5}$ i.e. $\mathrm{Zn}_{2} \mathrm{~S}_{2} \mathrm{C}_{4} \mathrm{~N}_{3} \mathrm{H}_{13}$ Calc. C, 16.12; H, 4.40; N, 14.10; Zn, 43.87; Exp. C, 18.84; H, 4.36, N, 13.57; Zn, 45.96.

\section{Characterisation}

Morphology of the $\mathrm{ZnS}$ nanosheets was investigated using various microscopy techniques. Samples were dispersed on aluminium stubs, sputter coated with gold and imaged on a Zeiss EVO SEM with an accelerating voltage of $10 \mathrm{kV}$. For TEM analysis suspensions were cast onto holey carbon-coated $3.1 \mathrm{~mm}$ copper grids and imaging was carried out on a JEOL 2011 TEM operated at $200 \mathrm{kV}$. The AFM images were obtained using a Nanoscope E AFM (scanner E) (Digital Instruments, Santa Barbara, USA) in Contact Mode in air. The sample for AFM imaging was prepared from a suspension of the crystals in ethanol and was drop-cast onto a freshly cleaved mica substrate, then imaged using a standard silicon nitride Contact Mode cantilever (type DNP-C, Veeco, USA) with a spring constant of $0.32 \mathrm{~N} / \mathrm{m}$.

Powder X-ray diffraction patterns were measured on a Bruker D8 Advance powder diffractometer at $40 \mathrm{kV}$ and $40 \mathrm{~mA}(\mathrm{Cu} \mathrm{K} \alpha$ with $\lambda=1.540607 \AA)$. Samples were step scanned from $5-90^{\circ} 2 \theta$ with a step size of $0.02^{\circ}$ and a counting time of 0.8 sec per step.

The thermal behaviour of the intercalated compounds was determined by thermogravimetric analysis using a Universal ${ }^{\circledR}$ V3.4C TA Instrument with a heating rate of $10^{\circ} \mathrm{C} \mathrm{min}^{-1}$, heated up to $600^{\circ} \mathrm{C}$ to constant weight in nitrogen flow.

\section{Results and Discussion}

The powder X-ray diffraction patterns of the two layered ZnS-amine products are shown in Figure 2. The pattern of the product obtained in ethylenediamine matches that described by Ouyang et al.[15] and displays the characteristic strong (200) reflection at $\mathrm{d}=8.63 \AA\left(10.24^{\circ} 2 \theta\right)$ which is in good agreement with the value of $8.627 \AA$ for the interlayer spacing between wurtzite $\mathrm{ZnS}$ sheets in $\mathrm{ZnS}(\mathrm{en})_{0.5}$. The pattern of the deta

product shows a strong peak at $d=12.30 \AA\left(7.18^{\circ} 2 \theta\right)$ indicating that this material also has a layered structure and that the interlayer spacing matches the dimensions of the 
larger diethylenetriamine molecule. The existence of a deta analogue of $\mathrm{Zn}(\mathrm{en})_{0.5}$ was inferred in the work of Wang et al[14], who prepared nanoscale wurtzite particles in a variety of morphologies using a solvothermal method in diethylenetriamine. However, these authors apparently did not isolate the ZnS-deta precursor phase and thus we present herein the first details of the isolation and characterisation of the $\mathrm{ZnS}($ deta) 0.5 hybrid composite.

The low-angle features in the XRD pattern of the product obtained in deta are of particular interest, as the very broad peak at $\sim 5.5^{\circ} 2 \theta$ suggests a strong similarity to the XRD patterns of certain clay minerals in which stacking of the layers is disordered.[18] In such minerals the occurrence of disordered stacking arrangements gives rise to a broad reflection at low angles, indicative of the variable intercalation. A likely explanation for this feature in the case of the $\mathrm{ZnS}$ (deta) intercalate is that the thickness of the wurtzite ZnS layers may be variable as demonstrated in Figure 3.

Figure 3 shows two possible schemes for the stacking of ZnS layers separated by intercalated diethylenetriamine molecules. In each of these schemes a single ZnS layer is represented as a solid sheet; each inorganic slab consisting of six-membered rings of alternating zinc and sulfur atoms such that it can be considered as a thin slice of the wurtzite ZnS structure. Each zinc atom is coordinated to three sulfur atoms within the inorganic layer, and one amine nitrogen atom on the organic linker. Scheme I shows the periodicity corresponding to $\mathrm{ZnS}$ (deta) 0.5 which gives rise the most intense reflection in the powder XRD pattern - the sharp peak at $7.18^{\circ} 2 \theta$ corresponding to $12.3 \AA$ between the ZnS layers. Scheme 2 demonstrates the disordered arrangement of alternating ZnS and diethylenetriamine layers that is believed to give rise to the broad reflection at $5.5^{\circ}$ 2 $\theta$. An irregular stacking arrangement of this type would also explain the observation of broad and poorly-resolved peaks in the higher angle portion of the pattern shown on the right in Figure 2. Note however, that amongst these broad signals, there is also a number of sharp reflections, many of them in positions corresponding to peaks observed for the $\mathrm{ZnS}(\mathrm{en})_{0.5}$ phase. This observation would seem to indicate that two distinct phases may be present in the sample.

Electron micrographs of the ZnS-amine layered composites are show in Figure 4 and the images shown in Figure $4 \mathrm{a}$ and $4 \mathrm{~b}$, provided here for the sake of comparison, demonstrate the remarkably regular rectangular platelets formed by the $\mathrm{ZnS}(\mathrm{en})_{0.5}$ layered 
composite phase. The SEM images shown in Figure 4c and 4d show that there are two distinct particle morphologies present in samples of the product formed in diethylenetriamine solvent. The larger particles have a similar platelet habit to the $\mathrm{ZnS}(\mathrm{en})_{0.5}$ particles, although without the regular straight edges and rectangular shape. Typical dimensions for these platelets were in the range of 1-5 $\mu \mathrm{m}$. Other particles in the sample appear to be rod-like and considerably smaller than the platelet particles, with typical dimensions of $\sim 1 \mu \mathrm{m}$ in length and about $100 \mathrm{~nm}$ in width. These images support the proposition that this sample is comprised of two distinct ZnS-deta composite phases. It seems reasonable to expect that the large platelets are particles of $\mathrm{ZnS}$ (deta) 0.5 while the smaller rod-like particles are a deta-deficient phase with a disordered stacking of ZnS layers, as described above.

TEM imaging of the $\mathrm{ZnS}(\mathrm{en})_{0.5}$ phase at moderate magnification provided a means of measuring typical thicknesses of the nanosheets, and an average thickness of $24 \mathrm{~nm}$ was established by locating several sheets that could be imaged from a side-on orientation. High resolution imaging of the layered composite phase was problematic however, as the particles were found to be very beam-sensitive, with regions of the platelets that were initially uniform rapidly becoming mottled in appearance under the effects of the electron beam. This effect is most likely due to phase transformation caused by localised heating of the particles, resulting in the loss of ethylenediamine from the structure. This behaviour has also been noted by Deng et al.[12] who described the rapid evolution of selected area diffraction patterns of $\mathrm{ZnS}(\mathrm{en})_{0.5}$ from a symmetrical spot pattern to polycrystalline rings within a short period of exposure in the electron beam.

In view of the problems associated with high resolution imaging of the platelets in the TEM, atomic force microscopy was considered as an ideal means to provide further insight into the surface nature of the platelets. AFM images of the $\mathrm{ZnS}(\mathrm{en})_{0.5}$ platelets are presented in Figure 5. The regular rectangular nature of the platelets is clearly demonstrated in the image presented in Figure 5a, but also notable is the truncated morphology of the corners of the sheets. The angles between edge faces consistently approximate to $45^{\circ}$ indicating the expression of small 011 faces at the corners of the platelets.

Although previous SEM and TEM images had suggested that the surfaces of the nanosheets were relatively smooth, high resolution imaging in the AFM revealed the 
presence of step features on the surface (Figure 5b). A line profile of a series of these step features is shown in Figure 5c, and it can be seen that the step heights match well with expected the $\sim 1.7 \mathrm{~nm}$ distance between (100) planes in the $\mathrm{ZnS}(\text { en })_{0.5}$ structure as described by Ouyang.[15] Thus we can conclude that the surface steps represent individual structural layers each composed of two wurtzite $\mathrm{ZnS}$ sheets and two intercalated layers of ethylenediamine molecules.

Thermogravimetric analysis of the two hybrid composites is presented in Figure 6 On heating to $650^{\circ} \mathrm{C}$ in an inert atmosphere, the ZnS(en) $)_{0.5}$ phase undergoes a sharp $23.4 \%$ endothermic weight lost event at $\sim 340{ }^{\circ} \mathrm{C}$, which corresponds to the removal of ethylenediamine from the compound, and results in collapse of the layered structure to yield wurtzite $\mathrm{ZnS}$. The same phase transition was reported in previous studies of $\mathrm{ZnS}(\mathrm{en})_{0.5}[12]$, and identical temperatures for removal of the diamine intercalate have been reported for $\mathrm{ZnSe}(\mathrm{en})_{0.5}$ and $\mathrm{ZnSe}(\mathrm{pda})_{0.5}[12,16]$. It is therefore quite surprising to observe that the ZnS-deta product does not show a similar weight loss event at $340^{\circ} \mathrm{C}$, but instead displays a two-stage weight loss process. Both weight loss events are exothermic: the first occurs at $\sim 180^{\circ} \mathrm{C}$ and the second is a less-sharply defined step over the temperature range of $350-450{ }^{\circ} \mathrm{C}$. The total weight loss is $35.6 \%$ which is reasonably close to the expected value of 34.6\%, although both elemental analysis and powder XRD analysis have suggested that the diethylenetriamine content of this material was slightly less than the stoichiometry of ZnS(deta) $)_{0.5}$. The TGA/DTA curves do provide further evidence that the product displays two different modes of binding of the diethylenetriamine intercalate, with one form that is more readily removed than the other. The fact that both of these weight loss events are exothermic, in contrast to the endothermic removal of diamine from other hybrid composites of this type, is surprising and is yet to be adequately explained. Studies of this material are ongoing and we hope to better explain the possible binding modes of intercalated diethylenetriamine through renewed attempts to isolate the discrete phases of ZnS-deta composites.

\section{Conclusions}

A new inorganic-organic hybrid material consisting of a semi-conducting layer of $\mathrm{ZnS}$ intercalated by organic diethylenetriamine has been synthesized, and found to contain a phase analogous to previously-described ZnS-diamine intercalates with the formula ZnS(diamine) $)_{0.5}$. Other ZnS-deta composities appear to be present in this 
material, in particular a phase in which the arrangement of diethylenetriamine and ZnS layers is disordered, with ZnS layers present in variable thicknesses.

The solvothermal formation of layered inorganic-organic hybrid composites based on wurtzite $\mathrm{ZnS}$ sheets with intercalated diamine molecules would appear to be achievable with a wide range of diamine molecules, but although such layered composites have been previously described with intercalated ethylenediamine and propylenediamine, the diethylenetriamine-based composite has been isolated and described here for the first time. The formation of a ZnS-deta layered hybrid composite with a structure analogous to $\mathrm{ZnS}(\mathrm{en})_{0.5}$ has been established from powder XRD results, but these also indicate that the product contains a deta-deficient layered phase in addition to the regular $\mathrm{ZnS}$ (deta) ${ }_{0.5}$ composite. The thermal analysis results and SEM images also indicate that two different types of ZnS-deta composite are present. Formation of more than one type of layered composite with diethylenetriamine is perhaps to be expected given that this molecule, having three amine groups rather than two terminal amines alone, could have various modes of binding to the wurtzite $\mathrm{ZnS}$ layers. The formation of pure $\mathrm{ZnS}(\text { deta })_{0.5}$, with the characteristic $12.3 \AA$ interlayer spacing would require that all diethylenetriamine molecules in the layer bind to $\mathrm{ZnS}$ layers only through the terminal amine groups. It is conceivable that, during lateral growth of a layered composite, binding of a diethylenetriamine molecule through the central, secondary amine moiety could result in termination of the regular layered structure and ultimately lead to the formation of "collapsed” ZnS layers. The series of mechanistic steps by which these layered composites grow, and the pathway for development of the unique rectangular plate morphology of these particles is not understood, but it is major goal of ongoing research. A detailed understanding of the factors dictating the formation of the layered structure may allow us to design and prepare inorganic-organic hybrid composites with finely controlled optical and electronic properties with a wide range of possible applications.

\section{Acknowledgements}

The authors wish to thank Dr Robert Hart for helpful discussions regarding analysis of XRD patterns. 


\section{FIGURES}

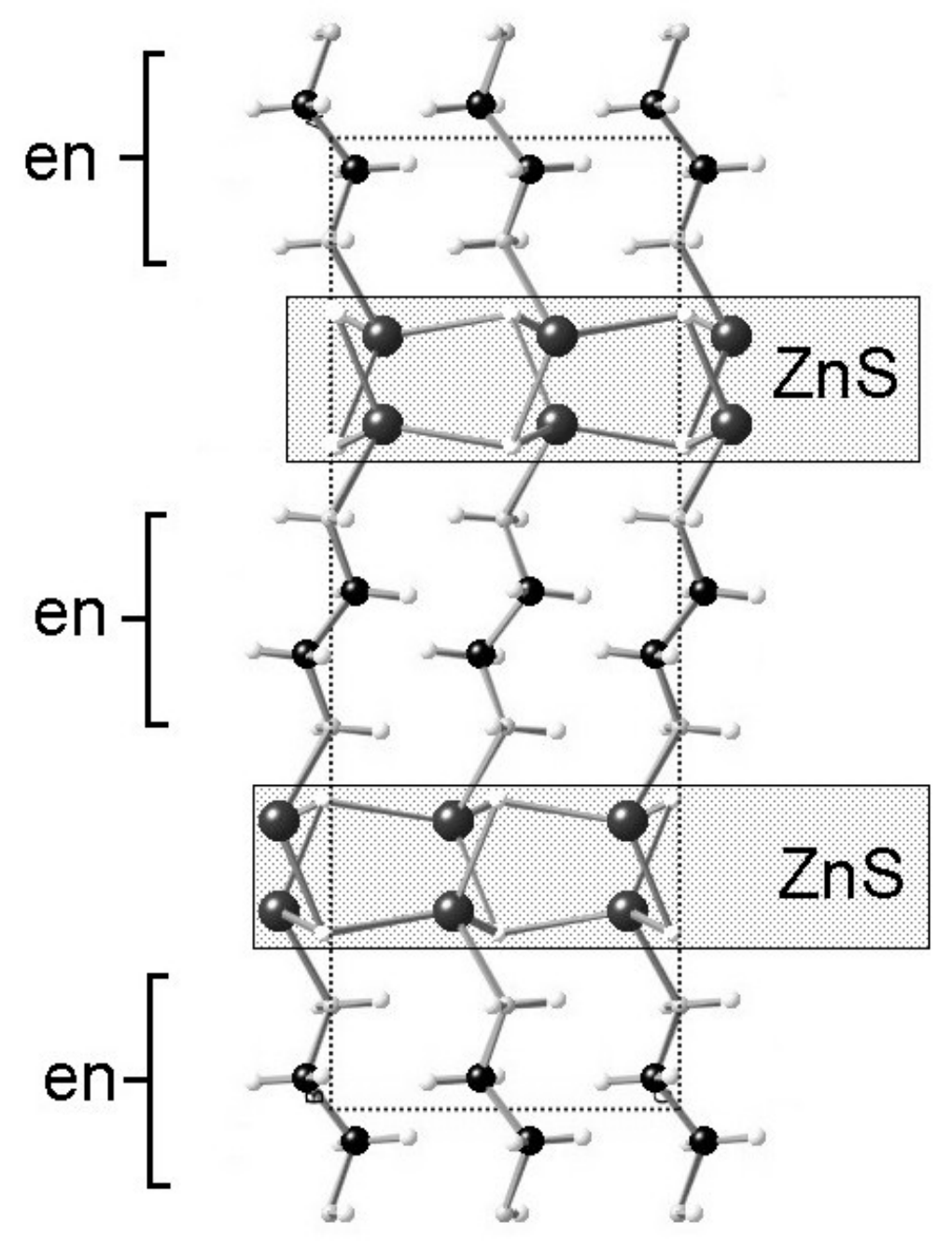

Figure 1: The structure of $\mathrm{ZnS}(\mathrm{en})_{0.5}[15]$ viewed along the y axis, showing alternating layers of wurtzite $\mathrm{ZnS}$ and linking ethylene diamine molecules. 


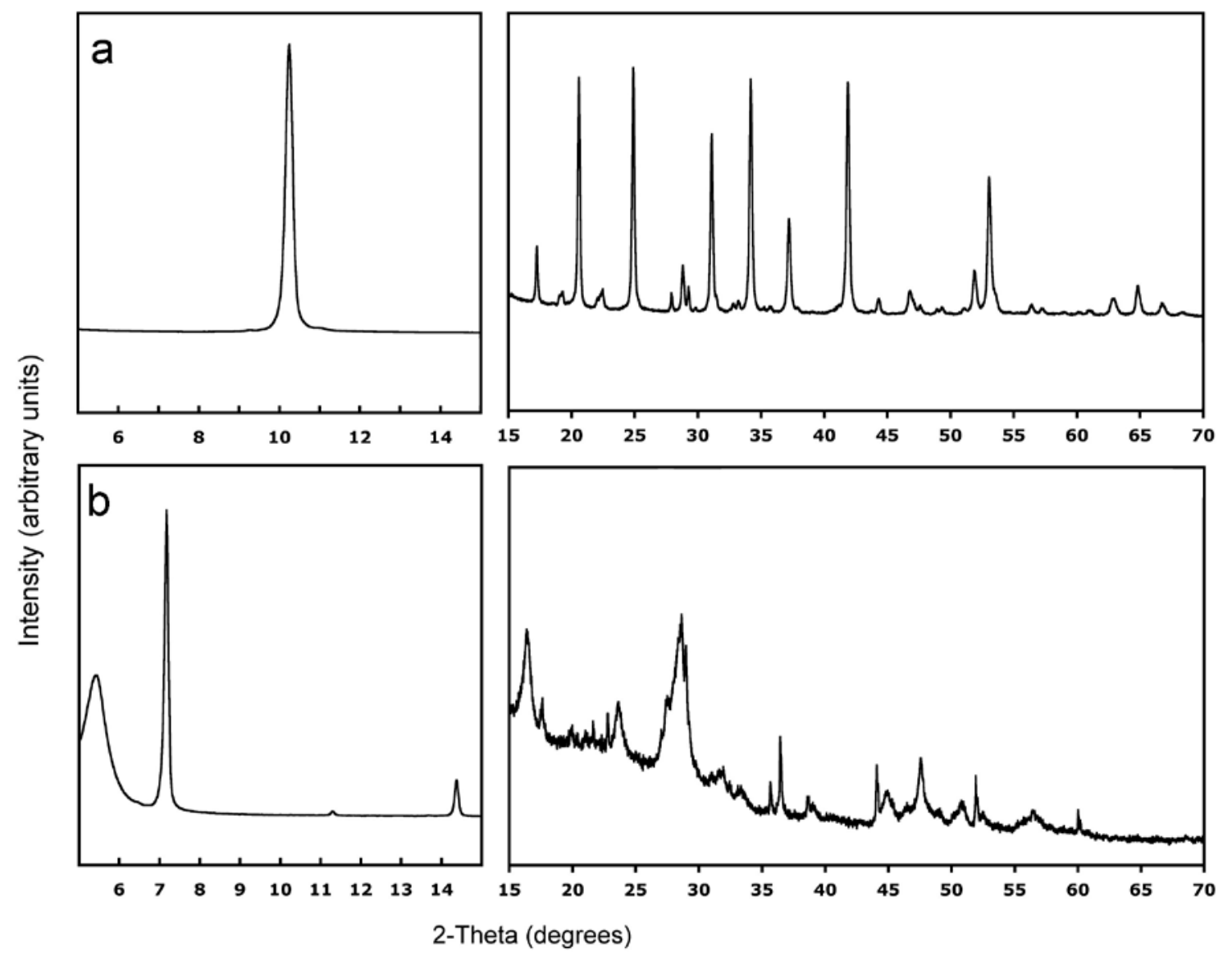

Figure 2: Powder XRD patterns of layered ZnS-amine composites. a) product obtained in ethylene diamine and (b) product obtained in diethylenetriamine. 

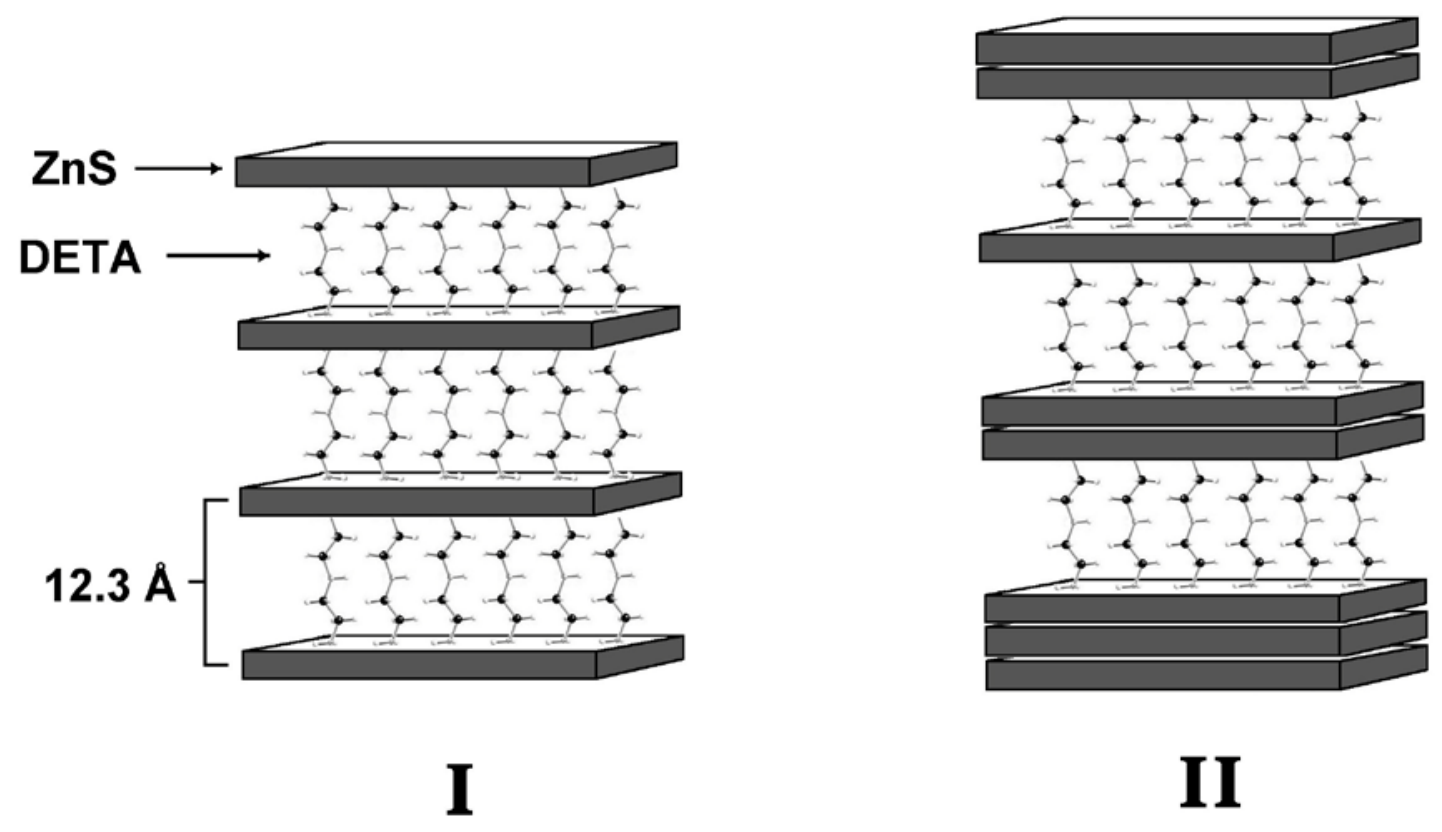

\section{II}

Figure 3: Two possible schemes for the arrangement of $\mathrm{ZnS}$ and diethylene triamine layers in a lamellar composite phase. Scheme I has the stoichiometry ZnS(DETA) 0.5 and is isostructural with $\mathrm{ZnS}(\mathrm{EN})_{0.5}$. Scheme 2 represents a disordered packing of $\mathrm{ZnS}$ layers, resulting in an irregular interlayer spacing. 

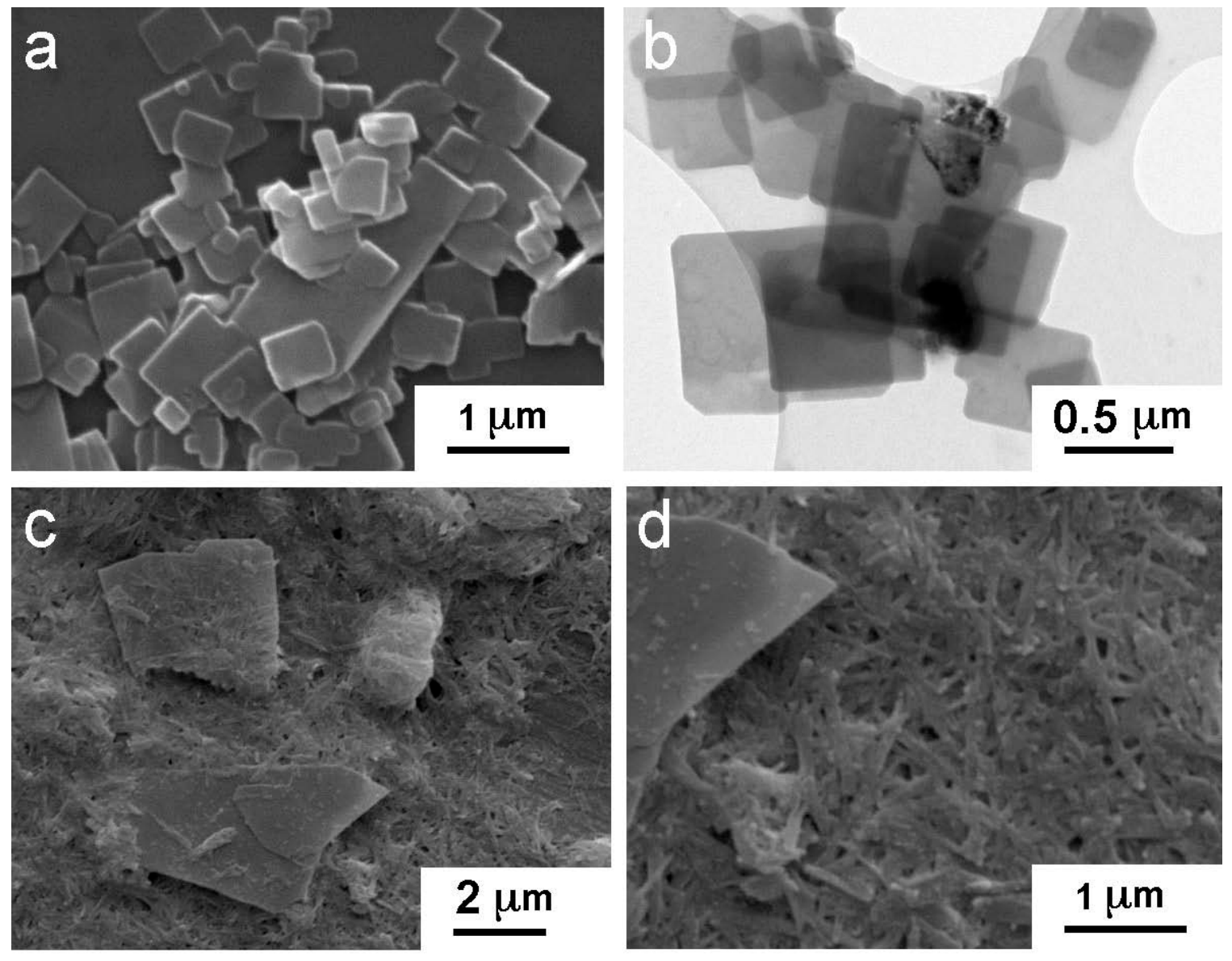

Figure 4: Electron micrographs of layered ZnS-amine composites. a) SEM image of ZnS(en) $)_{0.5}$ nanosheets; b) TEM image of ZnS(en) $)_{0.5}$ nanosheets; c) SEM image of the products obtained in diethylenetriamine showing both sheets and smaller rod-shaped particles. d) Higher magnification image showing rod-shaped particles. 

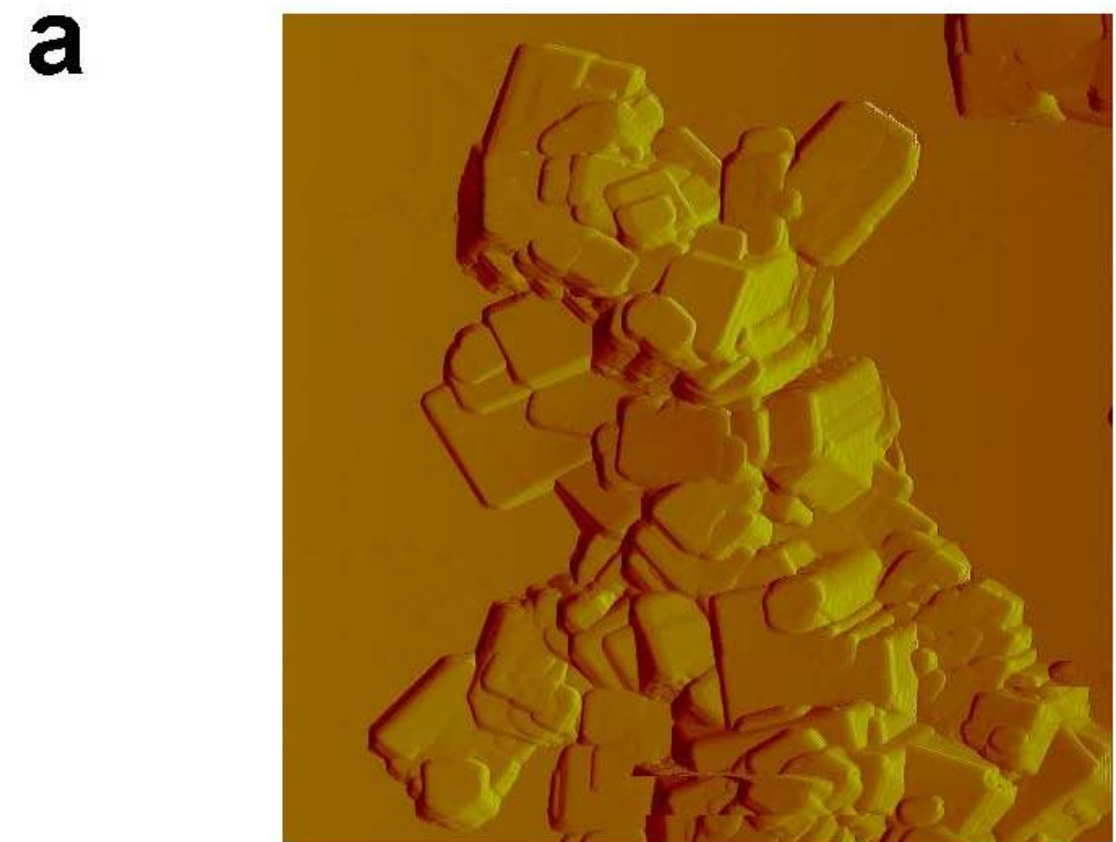

b
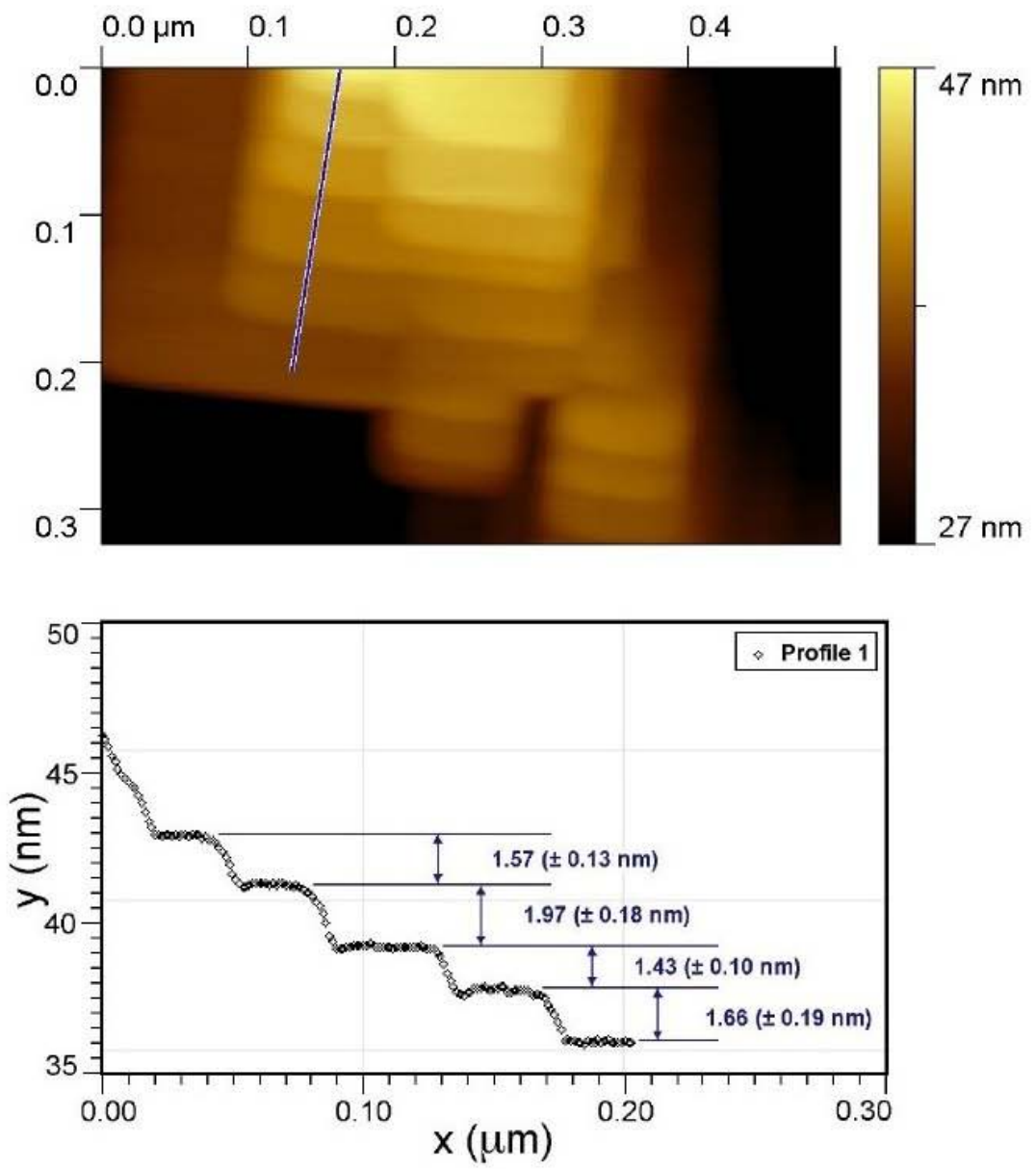

Figure 5: a) AFM image of ZnS(en) 0.5 platelets b) High resolution AFM image of the surface of a $\mathrm{ZnS}(\mathrm{en})_{0.5}$ nanosheet showing step-like features indicative of the layered structure of the sheets; c) Profile of the line trace drawn in image b showing the heights of step features on the surface. 

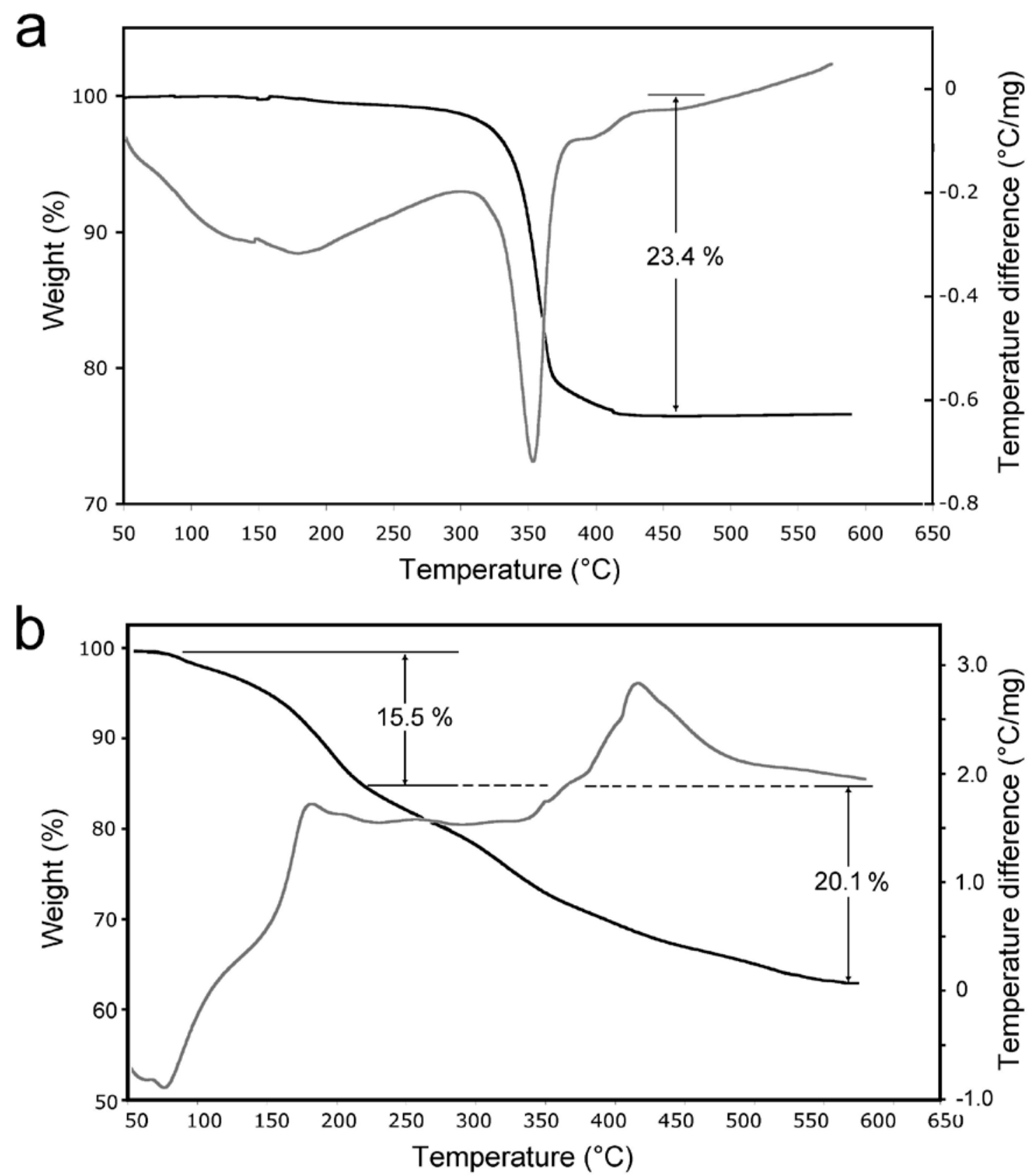

Figure 6: TGA/DTA curves of a) Zn(en $)_{0.5}$; b) The mixed-phase product obtained by solvothermal reaction diethylenetriamine. 


\section{References}

1. Kagan, C. R.; Mitzi, D. B.; Dimitriakolpoulos, C. D.: Organic-Inorganic hybrid materials as semi-conducting channels in thin-film field effect transistors. Science, 286, 945-947, (1999).

2. Huang, X.; Li, J.; Fu, H.: The first covalent organic-inorganic networks of hybrid chalcogenides: structures that may lead to a new type of quantum wells. J. Am. Chem. Soc., 122, 8789-8790, (2000).

3. Biswas, S.; Kar, S.: Fabrication of ZnS nonparticles and nanorods with cubic and hexagonal crystal structures: a simple solvothermal approach. Nanotechnology, 19, 045710, (2008).

4. $\quad$ Fang, X. S.; Ye, C. H.; Peng, X. S.; Wang, Y. H.; Wu, Y. C.; Zhang, L. D.: Largescale synthesis of $\mathrm{ZnS}$ nanosheets by the evaporation of $\mathrm{ZnS}$ nanpowders. J. Cryst. Growth, 263, 263-268, (2004).

5. Yue, G. H.; Yan, P. X.; Yan, D.; Liu, J. Z.; Qu, D. M.; Yang, Q.; Fan, X. Y.: Synthesis of two-dimensional micron sized single-crystalline $\mathrm{ZnS}$ thin nanosheets and their photoluminescent propereties. J. Cryst. Growth, 293, 428-432, (2006).

6. Kalyanikutty, K. P.; Gautam, U. K.; Rao, C. N. R.: Ultra-thin crystalline films of $\mathrm{ZnS}$ and PbS formed at the organic-aqueous interface. Solid State Sci., 8, 296-302, (2006).

7. Tang, H.; Xu, G.; Weng, L.; Pan, L.; Wang, L.: Luminescence and photophyscial properties of colloidal ZnS nanoparticles. Acta Materialia, 52, 1489-1494, (2004).

8. Gubin, S. P.; Kataeva, N. A.; Khomutov, G. B.: Promising avenues of research in nanoscience: chemistry of semiconductor nanoparticles. Russ. Chem. Bull., 54, 827-852, (2005).

9. Hamad, S.; Catlow, C. R. A.: Computational study of the relative stabilities of ZnS clusters, for sizes between 1 and 4 nm., J. Cryst. Growth, 294, 2-8, (2006).

10. Yu, S.-H.; Yang, J.; Qian, Y.-T.; Yoshimura, M.: Optical properties of ZnS nanosheets, ZnO densrites, and their lamellar precursor ZnS. Chem. Phys. Lett, 361, 362366, (2002).

11. Yu, S.-H.; Yoshimura, M.: Shape and phase control of ZnS Nanocrystals: template fabrication of wurtzite $\mathrm{ZnS}$ single-crystal nanosheets and $\mathrm{ZnO}$ flake-like dendrites from a lamellar molecular precursor $\mathrm{ZnS}\left(\mathrm{NH}_{2} \mathrm{CH}_{2} \mathrm{CH}_{2} \mathrm{NH}_{2}\right)_{0.5}$. Adv. Mater., 14, 296-300, (2002).

12. Deng, Z.-X.; Wang, C.; Sun, X.-M.; Li, Y.-D.: Structure-directing coordination template effect of ethylene diamine in formations of $\mathrm{ZnS}$ and $\mathrm{ZnSe}$ nanocrystallites via solvothermal route. Inorg. Chem., 41, 869-973, (2002).

13. Zhou, G.-T.; Wang, X.; Yu, J. C.: A low temperature and mild solvothermal route to the synthesis of wurtzite-type $\mathrm{ZnS}$ with single-crystalline nanoplate-like morphology. Cryst. Growth Des., 5, 1761-1765, (2005). 
14. Wang, X.; Zhu, Y.; Fan, H.; Zhang, M.; Xi, B.; Wang, H.; Qian, Y.: Growth of ZnS microfans and nanosheets: Controllable morphology and phase. J. Cryst. Growth, 310, 2525-2531, (2008).

15. Ouyang, X.; Tsai, T.-Y.; Chen, D.-H.; Huang, Q.-J.; Cheng, W.-H.; Clearfield, A.: Ab initio structure study from in-house powder diffraction of a novel $\mathrm{ZnS}$ (en)0.5 structure with layered wurtzite ZnS fragment. Chem. Commun., 886-887, (2003).

16. Huang, X.; Heulings, H. R.; Le, V.; Li, J.: Inorganic-organic hybrid composites containing MQ(II-VI) slabs: A new class of nanostructures with strong quantum confinement and periodic arrangement. Chem. Mater., 13, 3754-3759, (2001).

17. Heulings, H. R.; Huang, X.; Li, J.; Yuen, T.; Lin, C. L.: Mn-substituted inorganicorganic hybrid materials based on $\mathrm{ZnSe}$ : nanostructures that may lead to magnetic semiconductors with a strong quantum confinement effect. Nano Lett., 1, 521-525, (2001).

18. Moore, D. M.; Reynolds, R. C., X-ray diffraction and the identification and analysis of clay minerals. 2nd edition ed.; Oxford University Press: New York, 1997. 